\title{
Identification of a novel type of small molecule inhibitor against HIV-1
}

\author{
Byung Soo Kim ${ }^{1, \#}$, Jung Ae Park ${ }^{1 \#,}$, Min-Jung Kim ${ }^{1}$, Seon Hee Kim² ${ }^{2}$ Kyung Lee Yu $^{2}$, E Ji Chang You ${ }^{1,2, *}$ \\ ${ }^{1}$ Avixgen Inc., ${ }^{2}$ National Research Laboratory of Molecular Virology, Department of Pathology, School of Medicine, The Catholic University \\ of Korea, Seoul 137-701, Korea
}

\begin{abstract}
Here we report a new chemical inhibitor against HIV-1 with a novel structure and mode of action. The inhibitor, designated as A1836, inhibited HIV-1 replication and virus production with a $50 \%$ inhibitory concentration $\left(\mathrm{IC}_{50}\right)$ of $2.0 \mu \mathrm{M}$ in an MT-4 cell-based and cytopathic protection antiviral assay, while its $50 \%$ cytotoxic concentration $\left(\mathrm{CC}_{50}\right)$ was much higher than $50 \mu \mathrm{M}$. Examination of the effect of A1836 on in vitro HIV-1 reverse transcriptase (RT) and integrase showed that neither were molecular targets of A1836. The characterization and re-infection assay of the HIV-1 virions generated in the presence of A1836 showed that the synthesis of early RT products in the cells infected with the virions was inhibited dose-dependently, due in part to abnormal protein formation within the virions, thus resulting in an impaired infectivity. These results suggest that $\mathrm{A} 1836$ might be a novel candidate for the development of a new type of HIV-1 inhibitor. [BMB Reports 2015; 48(2): 121-126]
\end{abstract}

\section{INTRODUCTION}

Since human immunodeficiency virus type 1 (HIV-1), the causative agent of acquired immunodeficiency syndrome (AIDS), was first isolated, the development of antiviral drug has progressed rapidly and now more than 25 approved drugs are currently available to HIV-1 infected patients. Although many of these drugs target the viral enzymes, they can be categorized into different mechanistic classes. The majority of the approved drugs are reverse transcriptase (RT) inhibitors, which can be further subdivided into nonnucleoside RT inhibitors and nucleoside/nucleotide RT inhibitors (1). Another class of

*Corresponding author. Tel: +82-2-2258-7312; Fax: +82-2-22587790; E-mail: jiyou@catholic.ac.kr

${ }^{\text {"}}$ These authors contributed equally to this work.

http://dx.doi.org/10.5483/BMBRep.2015.48.2.239

Received 4 November 2014, Revised 14 November 2014, Accepted 18 November 2014

Keywords: HIV-1 inhibitor, Infectivity, Novel chemical structure, Reverse transcription, Viral protein processing
anti-HIV-1 drugs targets the HIV-1 protease (2). Additionally, a class of recently developed inhibitors blocks the activity of integrase, a viral enzyme required for the integration of the HIV-1 proviral DNA into the host DNA (3). Inhibitors of non-enzymatic targets, which inhibit the viral entry process either by blocking viral fusion or by acting as an antagonist against the host cell receptor CCR5 comprise an additional drug class (4). For the treatment of patients with HIV/AIDS, a so-called "HAART" (Highly Active AntiRetroviral Therapy) regimen, which consists of a combination of three or four different approved drugs, is being used currently due to the rapid emergence of single drug treatment regimen-resistant strains $(5,6)$. However, the emergence of drug resistant strains continues to present a daunting challenge, as strains resistant to a single drug often become cross-resistant to others due to the high mutational rate attributable to the intrinsic infidelity of HIV-1 RT (7). Such cross resistance can be severe as nearly all drug targets are affected by it, and thus HAART could no longer be an effective defense against newly generated strains. Therefore, the identification of new types of HIV-1 inhibitors that exhibit novel mechanisms of action and inhibit new targets is urgently warranted.

Toward the goal, efforts to identify new types of HIV-1 inhibitors against various other viral targets are currently being made. For example, DIBA and SAMT, acting as zinc chelators of the HIV-1 nucleocapsid protein that plays crucial roles during both the early and late stages of HIV-1 replication (8), have been identified and demonstrated anti-HIV-1 activity $(9,10)$. The numerous functions attributable to Vpr in the viral life cycle suggested that $\mathrm{Vpr}$ may also be a new HIV-1 drug target. Indeed, vipirin has been identified as an inhibitor of Vpr-dependent viral infection (11). Furthermore, BIT225, developed as a small molecule inhibitor of HIV-1 Vpu, was shown to exhibit antiviral activity against multiple drug-resistant strains of HIV-1 (12). An additional agent, RN-18, was identified as an antagonist of Vif function and showed inhibition of HIV-1 replication in APOBEC3G positive cells (13).

Here, we report the identification of a new type of small molecule anti-HIV-1 inhibitor, designated as A1836, with a novel structure that showed a potent anti-HIV-1 activity but little cellular toxicity. Results of virion characterization and in vitro viral enzyme assays indicated that the mode of action of

ISSN: 1976-670X (electronic edition)

Copyright (C) 2015 by the The Korean Society for Biochemistry and Molecular Biology

(c) This is an open-access article distributed under the terms of the Creative Commons Attribution Non-Commercial License (http://creativecommons.org/licenses/by-nc/3.0) which permits unrestricted non-commercial use, distribution, and reproduction in any medium, provided the original work is properly cited. 
A1836 was novel and different from that of well-known HIV-1 inhibitors that target viral enzymes such as RT or integrase. These results suggested that A1836 may be a good novel candidate for the development of a new type of HIV-1 inhibitor.

\section{RESULTS}

\section{Identification of a new anti-HIV small molecule inhibitor with a novel structure}

In our continuing endeavor to seek new anti-HIV small molecule inhibitors, we recently have identified a new chemical compound, designated as A1836 hereafter, with a novel structure (1-(4-chlorobenzyl)-N-(2-methoxybenzyl)-1 H-pyrazole-3-carboxamide) as shown in Fig. 1 using our cell-based antiviral assay, whose anti-HIV activity has never been reported previously.

\section{A1836 exhibited potent anti-HIV-1 activity but little cellular toxicity}

To examine the antiviral activity of A1836 against HIV-1, we first performed an antiviral assay using MT-4 cells infected with an HIV-1 NL4-3 isolate derivative carrying the enhanced green fluorescent protein (NL4-3EGFP) in the presence and absence of A1836. In this assay, the level of EGFP expression indicates the degree of viral infection and replication, as the NL4-3EGFP virus harbors an EGFP gene in the place of the viral Nef gene (14). Thus, the ability of a compound to inhibit the viral infection and replication within cells can be easily monitored by a simple observation of EGFP expression under a fluorescence microscope. As shown in Fig. 2A, treatment with the well-known HIV-1 RT inhibitors AZT ( $5 \mathrm{nM}$ ) or tenofovir $(1 \mu \mathrm{M})$ inhibited EGFP expression. Treatment with A1836 also exhibited a strong and dose-dependent inhibitory effect against HIV-1, as evidenced by a reduction in the EGFP expression level (Fig. 2A). When virus production was measured using an HIV-1 p24 antigen enzyme-linked immunosorbent assay (ELISA) with viral supernatants harvested from the antiviral assay, a potent and dose-dependent inhibition of virus production was observed (Fig. 2B). Based on this result, it was determined that $\mathrm{A} 1836$ had an anti-HIV activity with an $\mathrm{IC}_{50}$ of

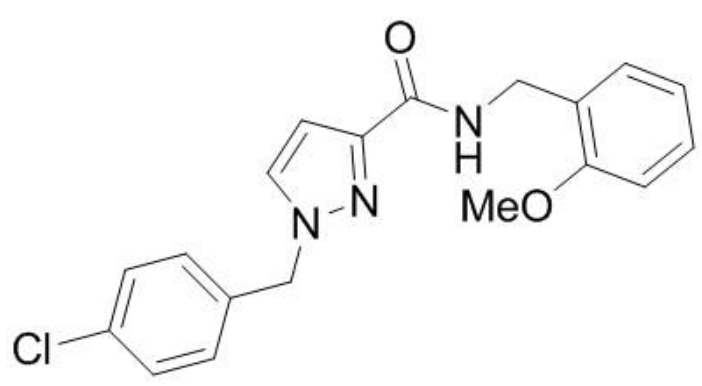

Fig. 1. Structure of A1836. Shown is the molecular structure of A1836, 1-(4-chlorobenzyl)-N-(2-methoxybenzyl)-1H-pyrazole-3-carboxamide.
2.0-2.5 $\mu \mathrm{M}$. Accordingly, further treatment with $5 \mu \mathrm{M}$ of A1836 inhibited nearly $90 \%$ of virus production (Fig. 2B). In the same assay, both AZT and tenoforvir also inhibited nearly $50 \%$ of virus production at $5 \mathrm{nM}$ and $1 \mu \mathrm{M}$, respectively, demonstrating the authenticity and reliability of this assay for anti-
$\mathbf{A}$

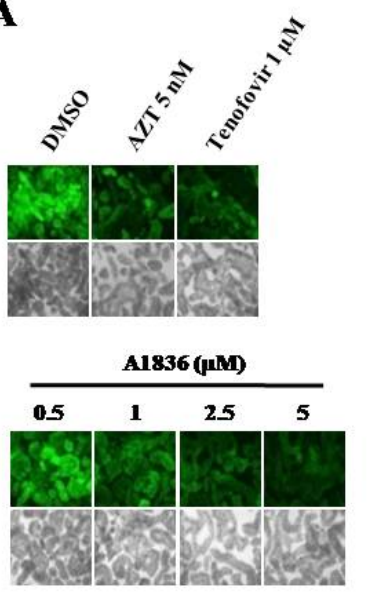

C

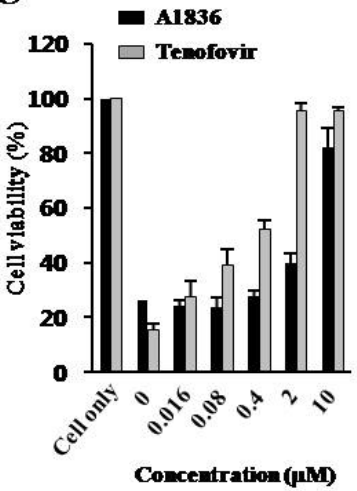

B

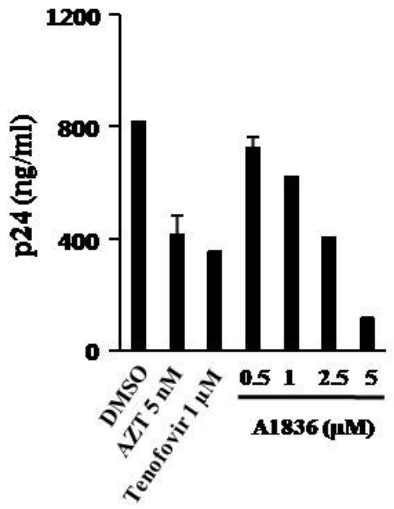

D

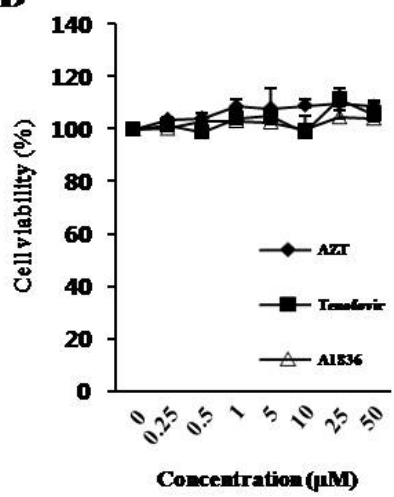

Fig. 2. The effect of A1836 on HIV-1 infection and replication. The anti-HIV-1 activity of A1836 was determined by both cellbased antiviral and cytopathic protection assays. MT-4 cells were infected with NL4-3EGFP, an HIV-1 NL4-3 isolate derivative, and treated with the indicated compounds. At 72 hours post-infection, the level of EGFP expression in the infected cells was observed by fluorescence microscopy (A) and viral supernatants were harvested and measured for the amount of virus production using an HIV-1 p24 antigen ELISA (B). (C) Determination of the HIV-1 cytopathic effect inhibition by A1836. MT-4 cells were infected with NL4-3GFP and treated with the indicated concentrations of A1836 and tenofovir as for a control. Following incubation for 5 days, cell viability was measured for each concentration using a cell viability assay. (D)The cellular cytotoxicity of A1836 was measured as described in the Material and Methods section. Data are presented as the percentage of cell viability in the presence of A1836 and indicated control inhibitors compared with that of non-treated cells. Data presented are the means \pm standard errors (SEs) of three independent measurements. 
viral efficacy measurement and determination. To further confirm the antiviral activity of A1836, we also employed an HIV-1cytopathic effect (CPE) inhibition assay (15) to determine whether A1836 had the ability to inhibit the cell death induced by HIV-1 infection, referred to as a cytopathic effect. To accomplish this, MT-4 cells were again infected with NL43EGFP and treated with various concentration of A1836 or tenoforvir as a positive control. After incubation for 5 days, cell viability was measured for each concentration by cell viability assay as described in the Materials and Methods section. As shown in Fig. 2C, tenofovir inhibited HIV-1 induced cell death in a dose-dependent manner, demonstrating the reliability of

$\mathbf{A}$

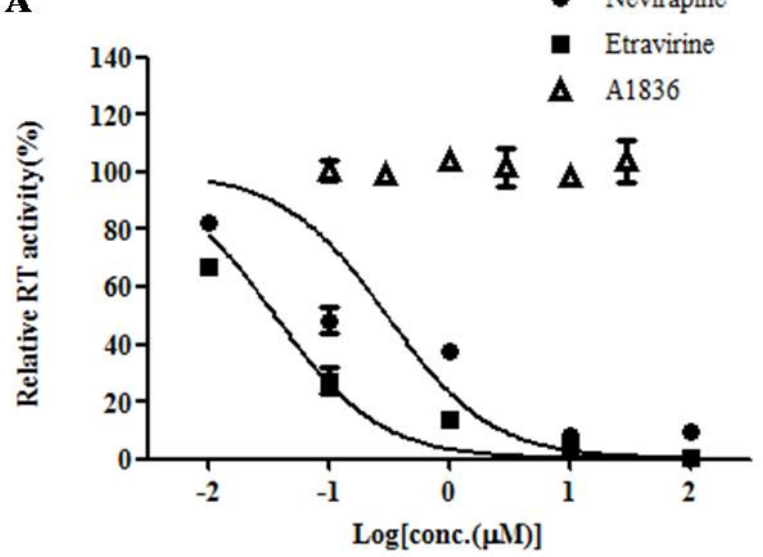

B

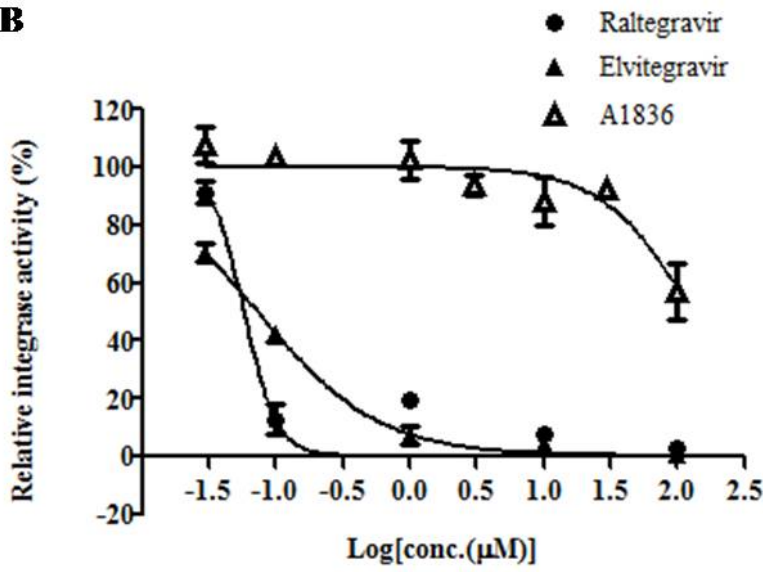

Fig. 3. The effect of $\mathrm{A} 1836$ on RT and integrase activity in vitro. (A) Determination of the effect of A1836 on RT activity in vitro. The effect of A1836 on HIV-1 RT activity in vitro was measured. Nevirapine and etravirine were used as positive controls. (B) Determination of the effect of A1836 on integrase activity in vitro. The effect of A1836 on integrase activity in vitro was measured as described in the Materials and Methods. Raltegravir and elvitegravir were used as positive controls. Data presented are the means \pm SEs of three independent measurements. this assay. The number of viable MT-4 cells was increased in an A1836 dose-dependent manner, clearly demonstrating that this new compound had anti-HIV-1 activity and the ability to inhibit the HIV-1-induced cytopathic effect.

To further confirm that the antiviral efficacy observed with A1836 was not due to cellular toxicity associated with use of the compound, we evaluated the cytotoxicity of A1836 on MT-4 cells. In this experiment, MT-4 cells alone were treated with $\mathrm{A} 1836$ from $0.25 \mu \mathrm{M}$ up to $50 \mu \mathrm{M}$ and incubated at $37^{\circ} \mathrm{C}$ for 3 days. Following incubation, cell viability was measured for each concentration by cell viability assay. The assay results demonstrated that A1836 showed no cellular toxicity as other control inhibitors AZT and tenofovir, even up to a concentration of $50 \mu \mathrm{M}$, indicating that $\mathrm{CC}_{50}$ of $\mathrm{A} 1836$ would be much higher than $50 \mu \mathrm{M}$ (Fig. 2D).

\section{Molecular targets of A1836-mediated inhibition of HIV-1 replication were neither HIV-1 RT nor integrase}

To investigate the molecular mechanism of the antiviral effect of A1836, we examined whether it could inhibit HIV-1 enzyme activity. First, the effect of A1836 against the activity of HIV-1 RT was determined using an RT assay in vitro. As positive controls, we employed the well-known RT inhibitors nevirapine and etravirine. Both nevirapine and etravirine inhibited HIV-1 RT activity in a dose-dependent manner, demonstrating the accuracy and reliability of this system (Fig. 3A). However, we did not detect any inhibition of RT activity in the presence of various concentration of A1836 up to $30 \mu \mathrm{M}$ (Fig. 3A). We also investigated whether A1836 could affect HIV-1 integrase activity. The effect of A1836 against the activity of HIV-1 integrase was measured using an HIV-1 integrase assay in vitro. In the assay, the well-known integrase inhibitors raltegravir and elvitegravir were used as positive controls. While raltegravir and elvitegravir inhibited HIV-1 integrase activity in a dose-dependent manner, no reduction of integrase activity by A1836 was detected (Fig. 3B). These results indicated that the molecular targets of A1836 that mediated the inhibition of HIV-1 replication were neither HIV-1 RT nor integrase.

Viral progeny generated in the presence of A1836 displayed impaired infectivity

To further elucidate possible targets and mechanisms of action attributable to A1836, we examined the endogenous reverse transcription activity of the virions generated in the presence of A1836 to determine whether intravirion reverse transcription activity was affected by A1836. The same amounts of viral particles produced in the antiviral assay depicted in Fig. 2A were incubated with reverse transcription reaction buffer as described in the Materials and Methods section in order to initiate endogenous reverse transcription. The concomitant viral DNA synthesized was then extracted and used for the quantitative polymerase chain reaction PCR (qPCR), performed using a primer pair within the $U 5$ and $R$ regions to assess the level of minus-strand strong stop DNA, an early product of reverse 
$\mathbf{A}$

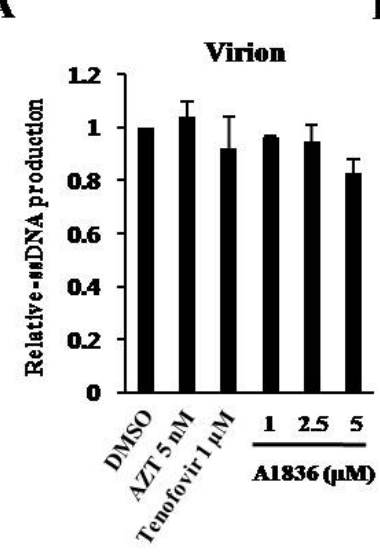

C

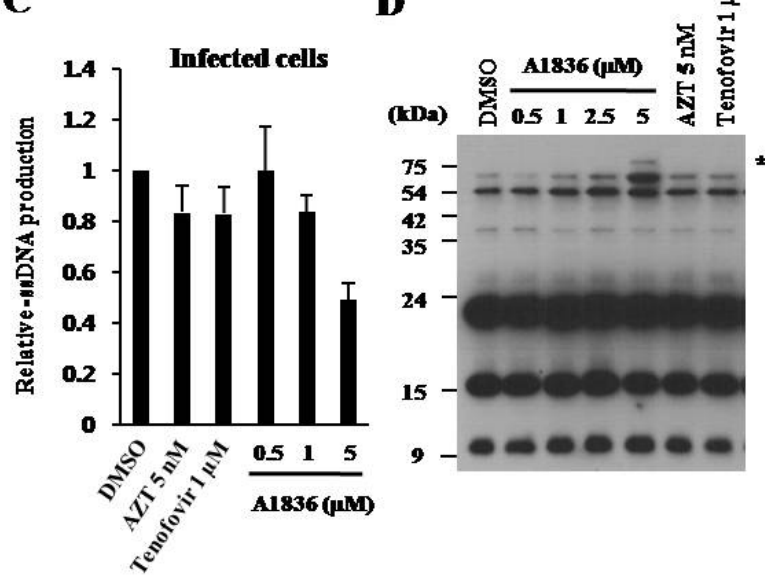

Fig. 4. The characterization of viral particles generated in the antiviral assay. (A) Measurement of intravirion reverse transcription in the viral progeny. The same amounts of viral particles produced in the antiviral assay depicted in Fig. 2A were subjected to an in vitro endogenous RT assay. A minus-strand strong stop DNA, an early RT product synthesized in viral particles, was measured using quantitative PCR as described in the Materials and Methods section. Data are expressed as the means \pm SEs of three independent experiments. (B) Impaired replication capacity of viruses produced in presence of A1836. M-T4 cells were infected with the same amounts of viral particles produced in the antiviral assay depicted in Fig. 2A, and EGFP expression in infected cells were observed at 72 hours post-infection by fluorescence microscopy (C) Measurement of intracellular reverse transcription activity of the viral progeny. MT-4 cells were exposed to the same amounts of viral particles from the antiviral assay and harvested at 6 hours postinfection. The level of early RT product synthesized within cells was measured using qPCR. Data are expressed as the means \pm SEs of three independent experiments. (D) Characterization of the protein content of the viral progeny. Viral particles produced in the antiviral assay depicted in Fig. 2A were analyzed by western

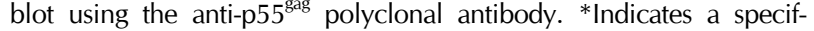
ic protein generated by A1836. transcription. As shown in Fig. 4A, minus-strand strong stop DNA was synthesized normally in all of the viral particles generated in the antiviral assay.

Next, we performed a re-infection assay to investigate whether viral particles generated in the presence of A1836 had the capacity for replication in the virus-infected target cells. When the same amounts of viral particles generated in the aforementioned antiviral assay were introduced to fresh MT-4 cells, we observed a dose-dependent decrease of the EGFP signal in cells infected with virions produced in the presence of the A1836 as compared to that of cells exposed to virions produced in the presence of other control HIV-1 inhibitors. These results suggested that the infectivity of the viral progeny produced in presence of A1836 was greatly impaired (Fig. 4B). To further determine quantitatively the properties of the observed impairment in the infectivity of virions generated in the presence of A1836, we analyzed whether the intracellular reverse transcription would be affected in infected cells. Thus, MT-4 cells infected with the same amounts of viral particles produced in the presence of A1836 were subjected to intracellular DNA extraction at 6 hours post-infection. The level of early RT product generated by the viral RT during viral infection was then measured by qPCR. As shown in Fig. 4C, the minus-strand strong stop DNA level was reduced in the cells exposed to virions produced in the presence of $5 \mu \mathrm{M}$ A1836 compared to that of cells exposed to virions produced in the presence of dimethyl sulfoxide (DMSO) or control HIV-1 inhibitors. These results indicated that the viral progeny generated in presence of A1836 had a replication defect and an inability to initiate reverse transcription in infected cells.

To further investigate the cause of the impaired infectivity of the viral progeny, we also characterized the viral proteins present in the viral particles generated in the antiviral assay by western blot analysis. As shown in Fig. 4D, similar amounts of Pr55gag and normally processed viral proteins were found in all of the viral particles generated in the antiviral assay. However, the dose-dependent increase of a higher molecularweight protein form of Gag (about $65 \mathrm{kDa}$ ) was observed in viral particles produced in the presence of A1836. Additionally, a protein corresponding about nearly $75 \mathrm{kDa}$ was also observed only in viral particles generated in the presence of 5 $\mu \mathrm{M}$ A1836. Thus, generation of abnormal protein forms in the viral progeny produced in the presence of A1836 correlated well with the reduction in the EGFP signal (Fig. 4B) in the re-infection assay and the inhibition of the intracellular RT product (Fig. 4C), suggesting that viral maturation or assembly may be a possible target of A1836 and underlie its observed anti-HIV activity.

\section{DISCUSSION}

In this study, we described a new candidate HIV-1 inhibitor, A1836, with a novel structure which restricted HIV-1 replication in a novel mechanism of action and the absence of a cel- 
lular cytotoxic effect. Treatment with A1836 resulted in a strong inhibition of HIV-1 replication (Fig. 2). However, further analysis determined that the key HIV-1 enzymes RT and integrase were not affected by A1836 (Fig. 3). Interestingly, when a re-infection assay was performed with viral particles produced in the antiviral assay, we found that viral progeny generated in the presence of A1836 had impaired infectivity (Fig. 4B). These viral progeny also showed a defect in their ability to initiate reverse transcription within infected MT-4 cells (Fig. 4C). When characterization of viral proteins present in viral progeny was performed, abnormal proteins were detected in viral particles generated only in the presence of A1836 (Fig. 4D). These results suggested that this new inhibitor did not affect the cellular host factor, but rather targeted the viral factors that operate during a late phase of the HIV-1 life cycle in which Gag protein maturation and assembly occur.

Formation of a highly ordered structure through proper Gag protein processing is essential and required for the generation of infectious particles and their replication, including proper activation of intracellular reverse transcription. When viruses with a hyperstable capsid structure, attributable to abnormal maturation and assembly, are infected into target cells, reverse transcription is prevented due to a delay in uncoating in the intracellular environment, while endogenous reverse transcription in virions occurs normally (16). A lower level of synthesized early RT products in the cells infected with virions with abnormal protein content, as shown in our results (Fig. 4), would account for the replication defect observed in the antiviral- and re-infection assays. Thus, the anti-HIV-1 property of this new inhibitor appears to be associated with its capability to generate viral progeny with impaired infectivity. Taken together, the observation implicate that the mode of action of A1836 is novel and different from that of well-known HIV-1 inhibitors and thus A1836 might complement the HAART to sustain further effectively the inhibition of HIV-1 replication. Although the specific viral targets of A1836 responsible for the generation of abnormal viral protein forms remain to be determined, these results clearly indicate that A1836 possesses highly potent anti-HIV-1 activity with either little or no cellular toxicity, and thus presents a good candidate for the development of a new type of anti-HIV-1 inhibitor.

\section{MATERIALS AND METHODS}

\section{Cell culture, virus preparation, and infection}

MT-4 cells were maintained in Roswell Park Memorial Institute medium (RPMI) containing 10\% fetal bovine serum (Hyclone, USA) and $1 \%$ penicillin/streptomycin. A recombinant HIV-1 virus expressing EGFP was obtained from 293FT cells transfected with a pNL4-3EGFP proviral plasmid. For the antiviral assay, MT-4 cells $\left(2 \times 10^{5}\right)$ were suspended in complete RPMI medium in 48 well plates, and NL4-3EGFP virus was added, with or without inhibitors, and incubated at $37^{\circ} \mathrm{C}$ for 3 days. After incubation, the levels of EGFP expression were measured by fluorescence microscopy (Olympus, Japan). Viral supernatants harvested from the antiviral assay were concentrated by centrifugation at $17,000 \mathrm{rpm}$ for 3 hours at $4^{\circ} \mathrm{C}$.

\section{HIV-1 p24 ELISA}

To measure the HIV-1 p24 titer in the viral supernatant, harvested viral supernatants were added to the 96-well plate provided with the HIV-1 p24 Antigen Capture Assay kit (Advanced Bioscience Laboratories, USA), and the viral titer was measured according to the manufacturer's instructions.

\section{HIV CPE inhibition assay}

MT-4 cells $\left(1 \times 10^{4}\right)$ were suspended in a complete RPMI medium in 96 well plates, and NL4-3GFP virus was added, with or without inhibitors, and incubated at $37^{\circ} \mathrm{C}$ for 5 days. After incubation, cell viability was determined for each concentration using a Cell-Titer Glo assay kit as described for the cell cytotoxicity assay.

\section{Cell cytotoxicity assay}

Cell cytotoxicity was measured with a Cell-Titer Glo assay kit (Promega Co., USA) according to the manufacturer's instructions. Briefly, MT-4 cells $\left(1 \times 10^{4}\right)$ were seeded on a white 96 well plate with compounds at concentrations ranging from 0 to $50 \mu \mathrm{M}$ and incubated at $37^{\circ} \mathrm{C}$ for 5 days. At the end of the incubation period, luminescence was measured with the SpectraMax Luminescence Microplate Reader (Molecular Devices, USA).

\section{HIV-1 RT assay}

HIV-1 RT activity was determined with an EnzChek Reverse Transcriptase Assay Kit (Invitrogen Co., USA) according to the manufacturer's instructions. Briefly, HIV-1 inhibitors were added to a reverse transcription reaction mixture containing HIV RTase (Abcam, USA), poly (A) templates, and oligo-dT and incubated at room temperature for 1 hour. The PicoGreen reagent provided with the kit was added and the resulting RNADNA duplexes were measured using a spectrofluorometer GEMINI EM system (Molecular Devices, USA).

\section{HIV-1 integrase assay}

HIV-1 integrase activity was determined with an HIV-1 integrase assay kit (XpressBio Life Science Products, USA) according to the manufacturer's instructions. The percentage of the inhibition of integrase activity was calculated by dividing the mean value in the presence of the tested antiviral compound with that in the presence of the DMSO control.

\section{Virion-associated endogenous- and intracellular reverse transcription}

For virion-associated endogenous reverse transcription, an equal amount of virions ( $1 \mathrm{ng}$ in p24) from the antiviral assay was incubated with an endogenous reverse transcription reaction buffer containing $0.01 \%$ Triton X-100, 50 mM Tris- $\mathrm{HCl}$ 
(pH 8.0), $50 \mathrm{mM} \mathrm{NaCl}, 10 \mathrm{mM} \mathrm{DTT}, 5 \mathrm{mM} \mathrm{MgCl}$, and 100 $\mu \mathrm{M} d N T P$ at $37^{\circ} \mathrm{C}$ for 2 hours. For intracellular reverse transcription, MT-4 cells $\left(1 \times 10^{5}\right)$ were infected with $20 \mathrm{ng}$ in p24 of virions from the antiviral assay and harvested at 6 hours post-infection. Viral DNA synthesized in virions and cells was extracted and analyzed as described for qPCR.

\section{DNA extraction and qPCR}

The viral DNA synthesized from virion-associated endogenous- and intracellular reverse transcription was extracted using the DNeasy Blood and Tissue Kit (Qiagen, Germany) according to the manufacturer's instructions. QPCR was then performed using a LightCycler480 (Roche Co., USA) and SYBR Green I Master mix (Roche Co., USA) with the following HIV-1 minus-strand strong stop DNA specific primers: Forward (5'-CAAGTA GTGTGTGCCCGTCTGTT-3'), reverse (5'-CTGCTAGAGATTTTTCCACACTGAC-3').

\section{Western blot analysis}

Virions were heat-denatured in sample buffer containing $8 \%$ sodium dodecyl sulphate (SDS), $250 \mathrm{mM}$ Tris- $\mathrm{HCl}(\mathrm{pH}$ 6.8), $40 \%$ glycerol, $0.02 \%$ bromophenol blue, and $5 \% \beta$-mercaptoethanol at $95^{\circ} \mathrm{C}$ for 10 minutes and analyzed by SDS-polyacrylamide gel electrophoresis. After transfer, the membrane was proved with anti-p55 antibodies (Thermo scientific, USA).

\section{ACKNOWLEDGEMENTS}

This work was supported by a grant of the Korea Healthcare technology R\&D Project, Ministry of Health \& Welfare (A121925) and in part by a research grant from the National Research Foundation of Korea (to J.C.Y.; 2013R1A2A2A01068353 and 2008-2004183).

\section{REFERENCES}

1. De Clercq E (1992) HIV inhibitors targeted at the reverse transcriptase. AIDS Res Hum Retroviruses 8, 119-134

2. Molla A, Granneman GR, Sun E and Kempf DJ (1998) Recent developments in HIV protease inhibitor therapy.
Antiviral Res 39, 1-23

3. Ingale KB and Bhatia MS (2011) HIV-1 integrase inhibitors: a review of their chemical development. Antivir Chem Chemother 22, 95-105

4. Ryser HJ and Flückiger R (2005) Progress in targeting HIV-1 entry. Drug Discov Today 10, 1085-1094

5. Mehellou Y and De Clercq E (2010) Twenty-six years of anti-HIV drug discovery: where do we stand and where do we go? J Med Chem 53, 521-538

6. Flexner C (2007) HIV drug development: the next 25 years. Nat Rev Drug Discov 6, 959-966

7. Condra JH, Schleif WA, Blahy OM et al (1995) In vivo emergence of HIV-1 variants resistant to multiple protease inhibitors. Nature 374, 569-571

8. Freed EO (1998) HIV-1 gag proteins: diverse functions in the virus life cycle. Virology 251, 1-15

9. Domagala JM, Bader JP, Gogliotti RD et al (1997) A new class of anti-HIV-1 agents targeted toward the nucleocapsid protein NCp7: the 2, 2'-dithiobisbenzamides. Bioorg Med Chem 5, 569-579

10. Srivastava P, Schito M, Fattah RJ et al (2004) Optimization of unique, uncharged thioesters as inhibitors of HIV replication. Bioorg Med Chem 12, 6437-6450

11. Ong EB, Watanabe N, Saito A et al (2011) Vipirin, a coumarin-based HIV-1 Vpr inhibitor, interacts with a hydrophobic region of VPR. J Biol Chem 286, 14049-14056

12. Dubé M, Bego MG, Paquay C and Cohen ÉA (2010) Modulation of HIV-1 host interaction: role of the Vpu accessory protein. Retrovirology 7, 144

13. Li ZY, Zhan P and Liu XY (2010) Progression in the study of HIV-1 Vif and related inhibitors. Yao Xue Xue Bao 45 684-693

14. Kwon HS, Park JA, Kim JH and You JC (2012) Identification of anti-HIV and anti-reverse transcriptase activity from Tetracera scandens. BMB Rep 45, 165-170

15. Hayashi S, Phadtare S, Zemlicka J, Matsukura M, Mitsuya $\mathrm{H}$ and Broder S (1988) Adenallene and cytallene: acyclic- nucleoside analogues that inhibit replication and cytopathic effect of human immunodeficiency virus in vitro. Proc Natl Acad Sci U S A 85, 6127-6131

16. Forshey BM, von Schwedler U, Sundquist WI and Aiken C (2002) Formation of a human immunodeficiency virus type 1 core of optimal stability is crucial for viral replication. J Virol 76, 5667-5677 